\title{
Comparing extremal and thermal Explorations of Energy Landscapes
}

\author{
Stefan Boettcher ${ }^{1, *}$ and Paolo Sibani ${ }^{2,0}$ \\ ${ }^{1}$ Physics Department, Emory University, Atlanta, Georgia 30322, USA \\ ${ }^{2}$ Theoretical Physics, Oxford University, 1 Keble Rd, Oxford OX1 3NP, UK
}

(Dated: September 21, 2018)

\begin{abstract}
Using a non-thermal local search, called Extremal Optimization (EO), in conjunction with a recently developed scheme for classifying the valley structure of complex systems, we analyze a shortrange spin glass. In comparison with earlier studies using a thermal algorithm with detailed balance, we determine which features of the landscape are algorithm dependent and which are inherently geometrical. Apparently a characteristic for any local search in complex energy landscapes, the time series of successive energy records found by EO also is characterized approximately by a log-Poisson statistics. Differences in the results provide additional insights into the performance of EO. In contrast with a thermal search, the extremal search visits dramatically higher energies while returning to more widely separated low-energy configurations. Two important properties of the energy landscape are independent of either algorithm: first, to find lower energy records, progressively higher energy barriers need to be overcome. Second, the Hamming distance between two consecutive low-energy records is linearly related to the height of the intervening barrier.

PACS number(s): 05.40.-a, 75.10.Nr, 02.60.Pn.
\end{abstract}

\section{INTRODUCTION}

The exploration of complex energy landscapes poses a series of problems of wide interest. Their multi-modal geometry is on one side challenging for optimization algorithms attempting to find the global minimum [1], while on the other side it provides a framework to model slow relaxation dynamics in nature [2]. Typically, in a physical (thermal) exploration of such relaxation phenomena, the static (geometric) and dynamic (algorithmic) aspects are intertwined. To disentangle the inherently geometric features, we apply a decidedly non-thermal optimization algorithm, called Extremal Optimization (EO) 1, 3, 4], to explore the energy landscape of a spin glass whose structure has been studied recently with a thermal algorithm [5]. Furthermore, the comparison with the thermal algorithm highlights distinct performance features of the EO algorithm.

Focusing on the temporal succession of energy values of record magnitude, we present a set of measures which characterizes the difficulty of local searches and the complexity of the landscape. There are at least two geometrical features of the spin glass landscape which are robust. For one, progressively higher energy states have to be surmounted in order to reach ever lower energy records. Second, a linear relation emerges between the Hamming distance of consecutive low-energy record configurations and the height of the highest intervening energy state. Such a relation has previously been found by other authors using different local search methods, as well as for other models [6, 7, 8]. Yet, in stark contrast to the thermal method, EO reaches a rapidly (exponentially) growing succession of high-energy records,

*Electronic address: www.physics.emory.edu/faculty/boettcher ${ }^{\dagger}$ Electronic address: Permanent Address: Fysisk Institut, SDU, Odensea $\$ \mathbb{K}$ O, uses a time series of unperturbed energy data. It projecting the search through configuration space by an exponentially growing Hamming distance between consecutive low-energy records.

In the following section, we describe how the landscape features explored by the dynamics can be assessed on the basis of a time series of suitably defined 'valleys'. In Sec. III we review the Extremal Optimization heuristic used here to produce such a time series for a non-thermal relaxation process. The main results of our numerical studies are discussed in Sec. [V] and in Sec. [V we present our conclusions.

\section{ENERGY VALLEYS IN COMPLEX LANDSCAPES}

The idea that progressively deeper, i.e. thermally more stable valleys are explored by the thermal dynamics of complex system is well-established and accounts for many important features of aging dynamics $[9,10,11,12,13,14,15,16,17,18]$. Qualitatively speaking, a valley would be a set of configurations sufficiently close to a local energy minimum. These configurations are repeatedly visited by the dynamics before the valley is "exited" and altogether different regions are explored. Ideally, in thermal systems the states within a valley would be visited with frequencies given by the respective Boltzmann weights, i.e. a local thermal equilibrium state is established before the valley is left.

The standard way of giving these concepts an operational meaning is to subject the dynamics to repeated thermal quenches, each leading to a local energy minimum or Intrinsic State (IS). The set of IS thus obtained partitions configuration space into basins of attraction with respect to thermal quenches, and each basin can be considered as a valley. A different approach [5], which we presently generalize to non-thermal algorithms such 


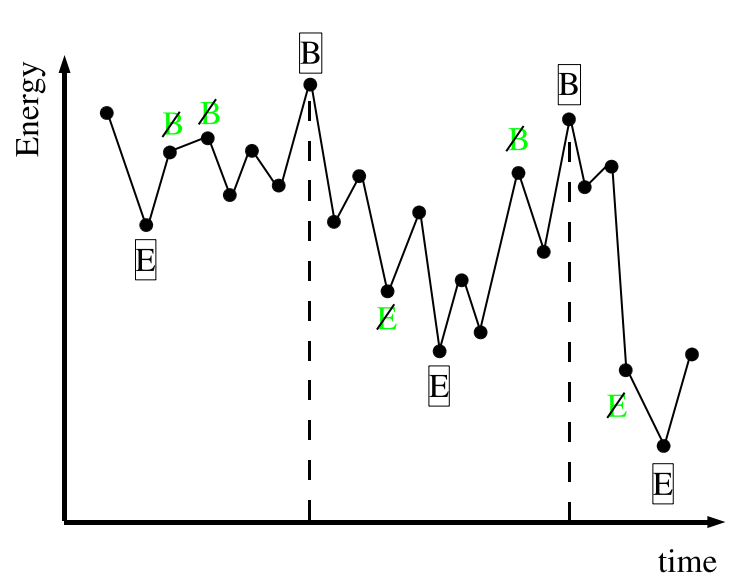

FIG. 1: The definition of a valley is illustrated with a fictitious series of energy values. A search produces a time sequence of energy (E) and barrier (B) records, where each "E" labels the lowest energy seen so far, and "B" refers to the highest barrier (relative to the most recent " $E$ ") reached up to that time. As explained in the text, in the end, only the highest barriers and the lowest energy records in each subsequence of "E"s and "B" $\mathrm{s}$ is kept, and the intermediate values are stricken from the record to give a strictly alternating sequence "EBEBE...". In particular, any two subsequent "B"s demarcate (entrance to and exit from) valleys, here separated by vertical dashed lines.

defines valleys on the basis of the lowest energy visited so far and is motivated by the fact that, for thermal dynamics, the dynamics is recurrent and equilibrium-like as long as this lowest energy state does not change.

As the details can be found in Ref. [5], only a brief account will be given for completeness (see Fig. 1): We keep track of the current lowest energy value $E$ encountered up to time $t$ and always measure the energy of the current state as the difference from this particular value. We furthermore keep track of the highest energy barrier $B$ visited, of the times at which both low and high records occur, and of the corresponding configurations. A trajectory is thus mapped into a symbolic sequence such as ...EEEBBBBBEEEEEEBBEEE ..., where the number of symbols in each subsequence of contiguous $E$ or $B$ values is larger or equal to one.

Consider first any subsequence of $E$ 's: since a trajectory 'sliding' downhill will produce such a sequence, all but the last $E$ correspond to transient states. By contrast, the last $E$ record may stand for a long time, i.e. at least until a record high energy value, the first subsequent $B$, is encountered. This makes it a good proxy for the lowest energy value in the 'current' valley. Similarly, while a trajectory explores high energy states it will likely visit several closely spaced energy maxima, producing a subsequence of $B$ values. The last $B$ value remains a record for long time, and is chosen to mark the passage from one valley to the next. In summary, all finite subsequences are pruned to their last element, producing a sequence ... BEBEBE ... where each triplet $B E B$ marks
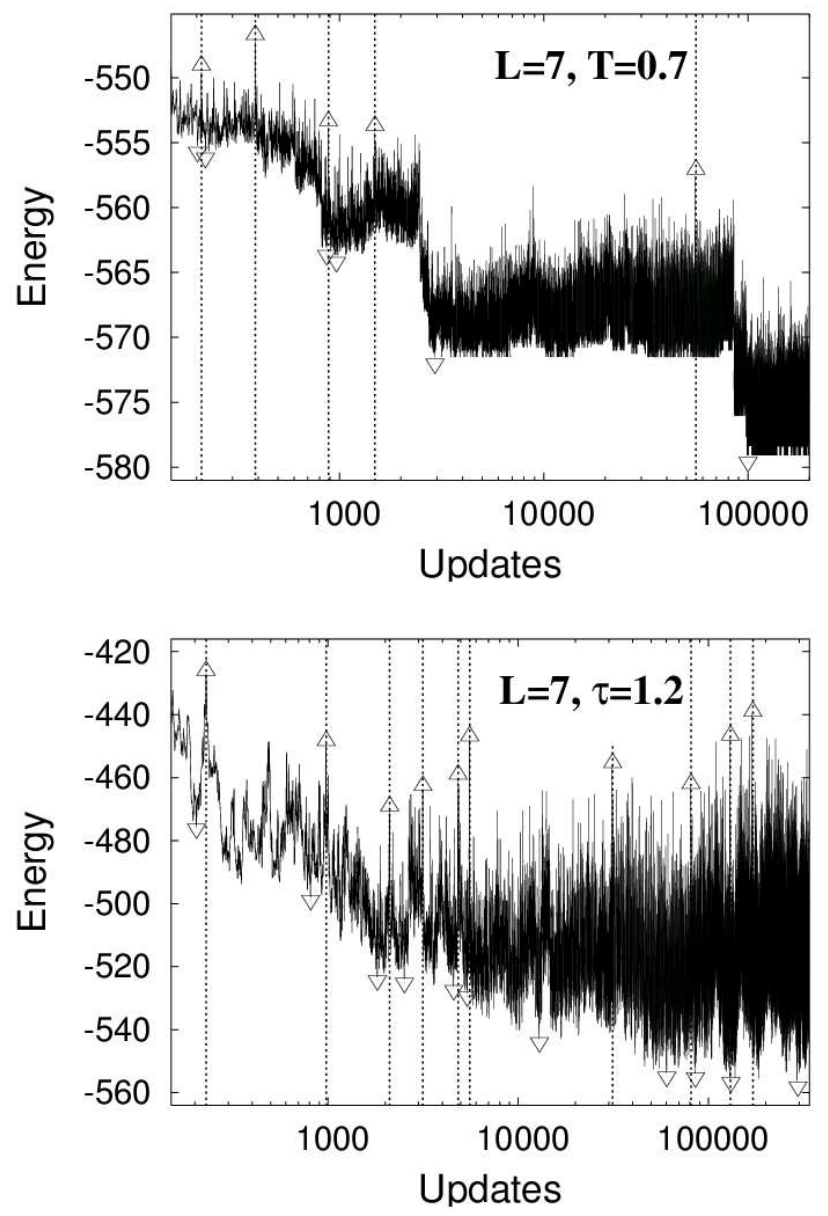

FIG. 2: Plot of a typical run with a thermal search (above) and an extremal search (below) for a $d=3$ Gaussian spin glass of size $L=7$. The fluctuating line marks the sequence of energies visited by the search. In terms of the definition in the text and Fig. 11 energy records (E) are marked by downtriangles, barrier records (B) by up-triangles. The barrier records also demarcate the beginning and the end of a valley, so each time interval between two consecutive vertical lines constitutes a valley. Counting valleys starts (with $n_{V}=0$ ) for updates $>N$ (where $N=7^{3}=343$ here) to avoid early transient behavior. While the absolute energy scale between both searches is not significant here (two distinct bond realizations were used), the difference in range and shape of the fluctuations is remarkable.

a valley, see Fig. 1 The $B$ s mark the enter and exit events and the the $E$ marks the lowest energy of the valley. The last sequence of $E$ 's or $B$ 's encountered in the simulation is not terminated and is discarded.

We note that this scheme produces trivial results (very few or no valleys, only infinitesimally increasing barriers, nearly same configurations for different $E$ 's) in cases where there is only one global minimum, or when the ground state can be chosen as starting point. Consider e.g. the case of a Metropolis random walk in a discrete set of energy value with a single minimum at energy zero. The downward drift implies that this minimum would 
most likely be reached without intervening barrier maxima, and the scheme will produce no output. In cases where a few 'false positives' are produced, i.e. valleys with no physical counterparts, these will closely resemble each other in terms of their configurations, energies etc. and will be immediately identified as such in the subsequent analysis. In any case, the time needed to reach the global minimum depends linearly, or in the lack of a bias, quadratically on the initial energy. The situation is completely different in complex energy landscapes, where, for thermal dynamics [5], and as shown below, for EO as well, the scheme gives a succinct but highly informative description of the dynamics: new valleys are accessed on a logarithmic time scale, and there is a systematic variation of their properties with the 'valley index'. We finally note that subsequent valley are defined on coarsening energy and time scales. Hence, each valley can be expected to contain many valleys of the previous kinds as sub-features. An illustration of the valley structure in a thermal and an extremal search is given in Fig. 2

From an optimization point of view, the $E$ values in the series represent the best the algorithm can do on a given time scale. The $B$ values act as energy barriers for a thermal type of algorithm, but not for an algorithm of the EO type, where energy differences have less dynamical significance. Nevertheless, if, as we expect, a geometric relationship links the Hamming distance between two "sufficiently low" minima and the height of the intervening barrier, the link should appear irrespective of the algorithm chosen. As shown below, the expectation is borne out by our simulations.

\section{EXTREMAL OPTIMIZATION}

An energy record statistic as described in the previous section can be generated by a variety of dynamical rules. Physically most relevant are those which evolve according to a thermal process that preserves detailed balance. The record statistics of a thermal process has been extensively studied previously for a number of different systems [5], including the Edwards-Anderson model with Gaussian couplings on a cubic lattice. A priori, it is not obvious which of the properties of this process can be attributed to the dynamic update rule, and which are inherently properties of the system.

A significant alteration of the update dynamics may in turn elucidate the origin of certain properties. To this end, we consider the Extremal Optimization (EO) heuristic [3, 4] as a decidedly different, athermal, update rule to explore the system. EO, like Simulated Annealing (SA) [19, 20], attempts to advance toward lower energy values via a local search of the landscape. Unlike SA, EO is modeled after driven dissipative processes, intentionally pushing the dynamics away from local equilibrium and detailed balance.

While a distinction between static landscape and dynamic properties is desirable in its own right, the simi- larity of these physical systems to many practical combinatorial optimization problems in computer science provides additional incentive for a broad-based investigation of local search methods and their ability to exploit the landscape geometry. EO in particular has proved to be a competitive heuristic to determine low-energy configuration for some of the hardest combinatorial optimization problems known, including graph bipartitioning 21], coloring [4, 22], and also spin glass problems 23]. We can hope that a more detailed view at the interplay of heuristic search and landscape geometry will lead to improvements in the quality of the results found as well as in the speed of convergence.

The extremal optimization algorithm, $\tau$-EO, which we employ in this paper, has been discussed previously in [4], and in [24, 25] with regard to the setting of its sole free parameter, $\tau$. At each instant during the search of a particular instance, $\tau$-EO assigns to each spin $x_{i}$ in the configuration its contribution to the total energy as "fitness,"

$$
\lambda_{i}=\frac{1}{2} x_{i} \sum_{<, j>} J_{i, j} x_{j}-\frac{1}{2} \sum_{<, j>}\left|J_{i, j}\right|,
$$

where the summation extends over all neighboring spins $x_{j}$ of $x_{i}$. Note that the second term on the right corresponds to the (absolute) weight attributable to that spin; it ensures that for each variable its optimal fitness is zero, irrespective of its overall weight. Accordingly,

$$
H=-\sum_{i} \lambda_{i}-\sum \sum_{<i, j>}\left|J_{i, j}\right|,
$$

i. e. the sum of all fitnesses tallies the total energy, aside from a trivial offset.

During a search with $\tau$-EO, we rank all $x_{i}$ according to fitness $\lambda_{i}$, i.e., we find a permutation $\Pi$ of the variable labels $i$ with

$$
\lambda_{\Pi(1)} \leq \lambda_{\Pi(2)} \leq \ldots \leq \lambda_{\Pi(n)} .
$$

The variable $x_{j}$ with the worst burden on the total energy is of rank $1, j=\Pi(1)$, and the best variable is of rank $n$. Consider a scale-free probability distribution over the ranks $k$,

$$
P_{k} \propto k^{-\tau}, \quad 1 \leq k \leq n,
$$

for a fixed value of $\tau$. At each update, select a rank $k$ according to $P_{k}$. The spin $x_{j}$ with $j=\Pi(k)$ is forced unconditionally to change state. For $\tau>0$ this selection process ensures a certain preference in fixing the state of spin variables which put a higher burden on the total energy. In particular, it has been found that intermediate choices for the value of $\tau$ in Eq. (4), with $\tau-1 \sim 1 / \ln (n)$ [24], often lead to the best results for a given runtime of the algorithm. Values of $\tau$ much larger or smaller than these quickly produce too confined or too random searches, as we will see. This issue has been explored also in Ref. [26]. 
The definition of fitness, which generally permits variations that can significantly impact performance 3], as given in Eq. (1) is purely a measure of "badness" in each variable. The constant offset in each $\lambda_{i}$ in Eq. (1), consisting of the absolute weight of all attached bonds, ensures that perfectly well adapted spins, $i$. e. those bordering on bonds that are all satisfied, have zero fitness, irrespective of their overall weight. Spins bordering on unsatisfied bonds get penalized according to their burden on the total energy. Considering that the absolute sum of all weights associated with spins is distributed unevenly, heavy-weighted spins (and bonds) are satisfied with a higher priority. At later stages of the search, the overwhelming number of weaker bonds are attended to.

\section{NUMERICAL RESULTS}

In this section, we present the results of extensive numerical investigations of the $\tau$-EO search for the $d=3$ Edwards-Anderson spin glass. The procedure, as outlined in Sec. II] follows closely that of Ref. [5]. There, a rejection free implementation of the Metropolis algorithm, the Waiting Time Method (WTM) 27], was used to study the thermal dynamics of this spin glass at low temperatures starting from a hard quench. In the process, many salient features of the record statistics have been measured, which we will focus on also in this study.

We run the $\tau$-EO search for a large number of instances of varying lattice size to explore the finite-size scaling properties of the observables with sufficient accuracy. In particular, we have used from 100,000 bond realizations for $L=8$ to 1,000 such instances for $L=20$ and performed 3 runs each. As in the thermal process, each run starts from random initial conditions in the spin configuration. A form of local "equilibration" is reached within a few EO-sweeps of the system, during which each spin gets to arrange itself with the local field imposed by its neighbors. After one sweep, we start sampling low-energy and barrier records through a sequence of valleys. In particular, we run the EO algorithm on each instance for $O\left(N^{2}\right)$ update steps, where $N=L^{3}$, or about $10 N$ sweeps of the system. Here, a "sweep" refers to $N$ EO-update steps, which are stochastic and do not imply that each variable is updated exactly once. Note that the valley index is gauged to be $n_{V}=0$ after the first $(t=1)$ sweep of the system.

\section{A. Varying $\tau$}

First, we have studied the dependence of EO's performance on the parameter $\tau$. To this end, we have conducted about 10,000 runs of EO on random instances at fixed system size $L=16$ for $\tau=0.2,0.7,1.2,1.7$, and 2.2. The behavior of EO has previously been show to be very sensitive to this parameter. Overall, we notice that, indeed, the results of the record statistics are as

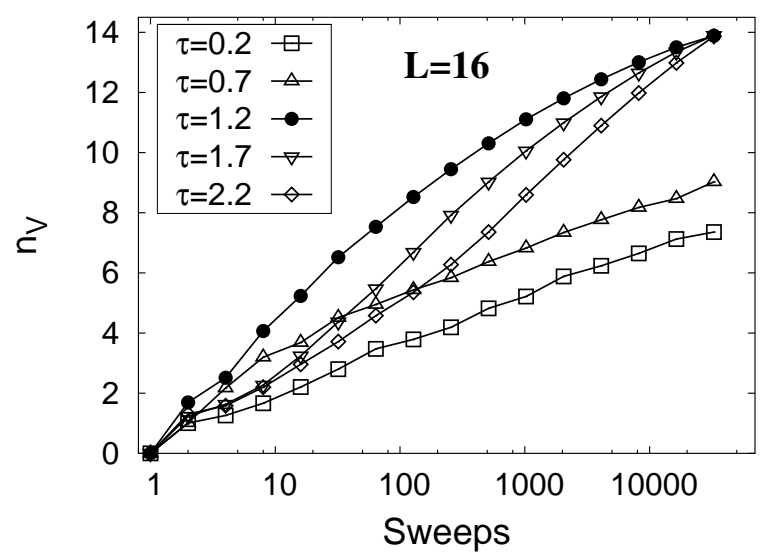

FIG. 3: Plot of the valleys found by EO on a logarithmic timescale for a system of $L=16$ but with values of $\tau=0.2$, $0.7,1.2,1.7$, and 2.2. The exploration of new valleys occurs at a much faster rate for intermediate values of $\tau$, until at later times saturation due to finite size effects permits runs with higher $\tau$ to catch up.

well strongly $\tau$-dependent. This dependence is often not monotone in $\tau$.

Most significantly, as Fig. 3. shows, the most valleys, and the best energy records, are obtained at intermediate values of $\tau$, i. e. those valleys are found on much shorter timescales than for $\tau$ values that are too large or too small. Since there is a rapid gain in new valleys for $\tau=1.2$, saturation effects due to finite system size set in faster, a trend to which higher values of $\tau$ eventually catch up. Overall, there is a more pronounced variation with the parameter $\tau$ than in the corresponding data for a thermal search (see Fig. 1 in Ref. 5]). From an optimization standpoint, $\tau$-EO progresses through these valleys with about ten times fewer sweeps than a fixedtemperature search.

The trend that an intermediate value of $\tau$ provides the favorable search results is also reflected in the energy records found in those valleys, as Fig. 4 shows. Thus, for an "optimal" value of $\tau$ near unity, EO finds new valleys faster and the energy states accessed within those valleys are lower. In fact, the exploration for larger values of $\tau$ proves qualitatively similar, just on a slower timescale. The behavior for $\tau<1$ is quite distinct, more akin to a high-temperature thermal diffusion. These observations are consistent with the phase transition at $\tau=1$ in the search dynamics of $\tau$-EO found for a model problem in Ref. 24].

At low values of $\tau$ the search is too random, and whatever valleys are found are not explored with sufficient "greed." This randomness expresses itself also in very high energy states accessed in between valleys, and the total decorrelation between successive valleys as illustrated by the large Hamming distances between the consecutive records in energy. (Hamming distance refers to the number of spin flips that separates any two configurations.) These features are displayed in Figs. 5 and 6 . 


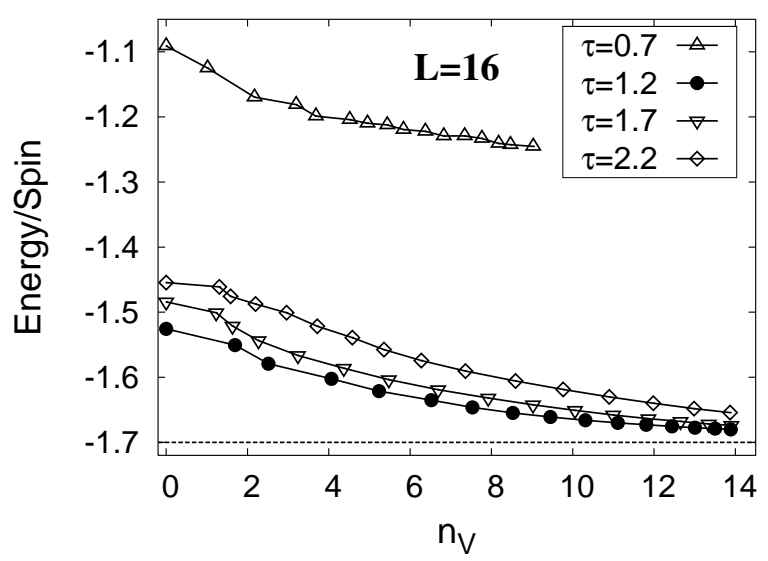

FIG. 4: Plot of the energy records found by EO within a given valley as a function of the valley index $n_{V}$. Not only do we find new valleys faster at intermediate $\tau \approx 1.2$ (as Fig. 3 shows), but also are lower energy records found within, even at equalized valley index $n_{V}$. (For $n \rightarrow \infty$, average ground state energies are $\approx-1.70[28]$.) Note the dramatic shift between $\tau=0.7$ and $\tau=1.2$. (Data for $\tau=0.2$ is even further above that for $\tau=0.7$ and has been dropped here.)

in which the barrier records and Hamming distances of consecutive energy records are plotted for each valley index. These properties are quite monotone in $\tau$ as can be expected from the nature of the optimal EO search as a compromise between too random and too greedy behavior [24, 25]. Hence, on the greedy side, for larger $\tau$, EO spends a long time in each valley, while reaching down to very low-energy states within each valley, before escaping through an equally low barrier, which only provides access to a new valley with states highly correlated (small Hamming distance) with those in the previous one. Conversely, for smaller $\tau$, the search approaches a random walk through the configuration space that is unlikely to reach down to very low-energy states. Any memory of fit variables in the list in Eq. (3) is short-lived, and the search trajectory quickly decorrelates such that the Hamming distances between consecutive barrier records soon saturate at the system size $(n / 2)$, see Fig. [6]

Most surprising is the rapid increase of both, barrier heights and Hamming distances, which scale exponentially with the valley index for sufficiently large values of $\tau$. This is in marked difference with the behavior of the thermal relaxation, where both grow about linearly with the index (see Figs. 2 and 3 in Ref. [5]). In contrast, near $\tau=0$ (which rigorously corresponds to $T=\infty$ ) we recover the linear scaling of the barrier heights with valley index observed for a thermal search, as the upper plot in Fig. [5demonstrates, again a hint of the phase transition at $\tau=1$. In general, barrier heights and Hamming distances vary significantly for both, extremal and thermal exploration, with their respective parameters.

Despite this difference between extremal and thermal exploration, in both cases the scaling of the Hamming distance itself with the barrier, as shown in Fig. 7 is
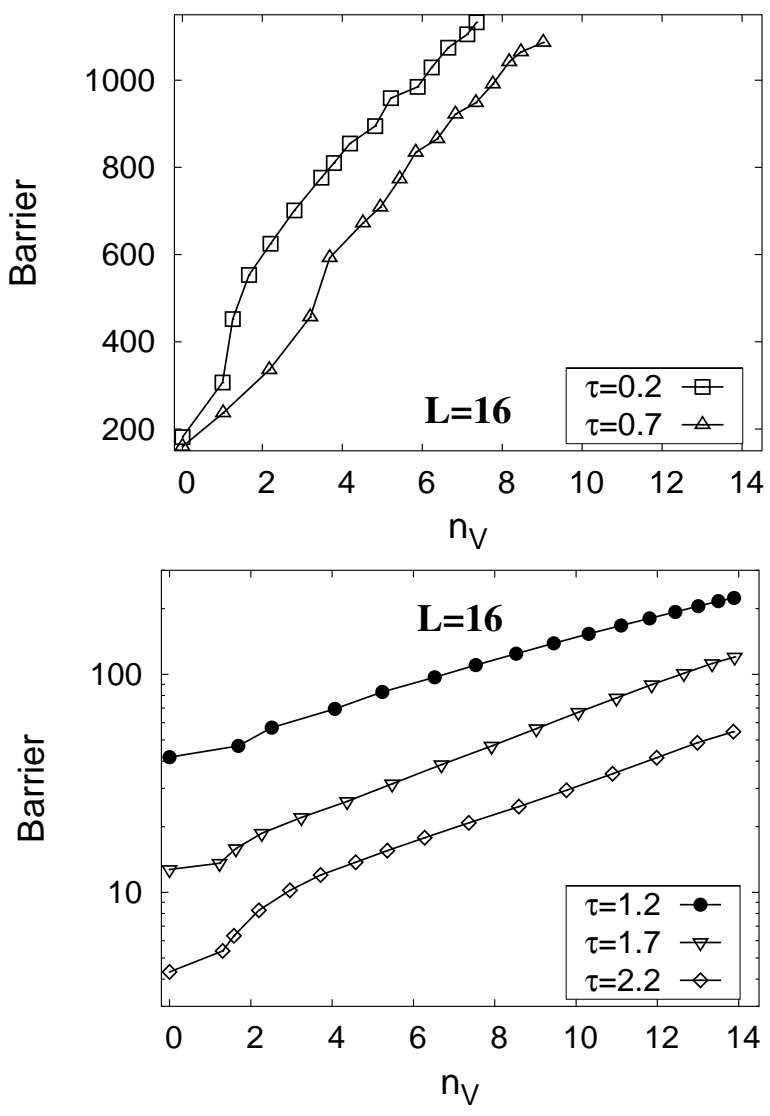

FIG. 5: Plot of the highest energy level (or barrier) accessed between two successive low-energy records as a function of valley index $n_{V}$. (Note that the height is measured relative to the most recent low-energy record.) Unlike in Figs. 3 and 4 the $\tau$-dependence here is monotone. Yet, for $\tau<1$ (upper Figure) the barrier height varies only linearly with the valley index, similar to a thermal search, while for $\tau>1$ (lower Figure) barrier heights rise exponentially.

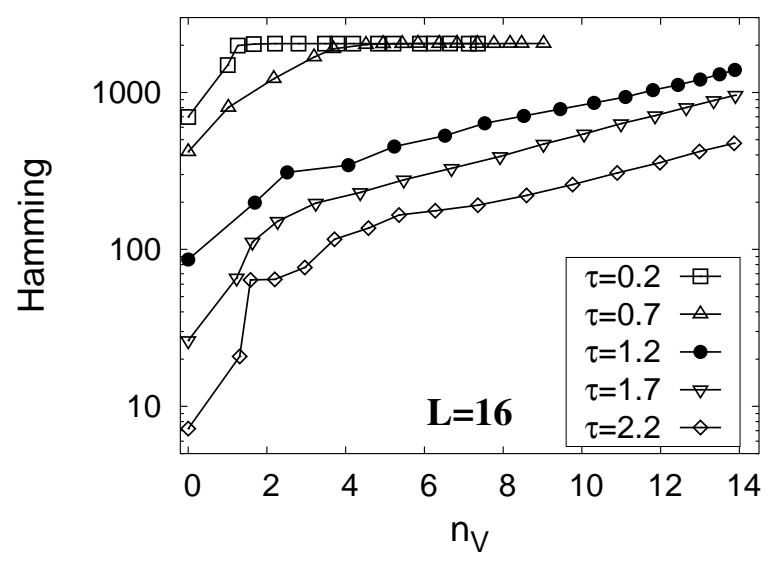

FIG. 6: Plot of the Hamming distance between successive low-energy records as a function of valley index $n_{V}$. As in Fig. 5 the $\tau$-dependence here is monotone, showing that the search gets increasingly narrow for increasing $\tau$. For small $\tau$, the Hamming distance quickly saturates at the system size $(n / 2)$. 


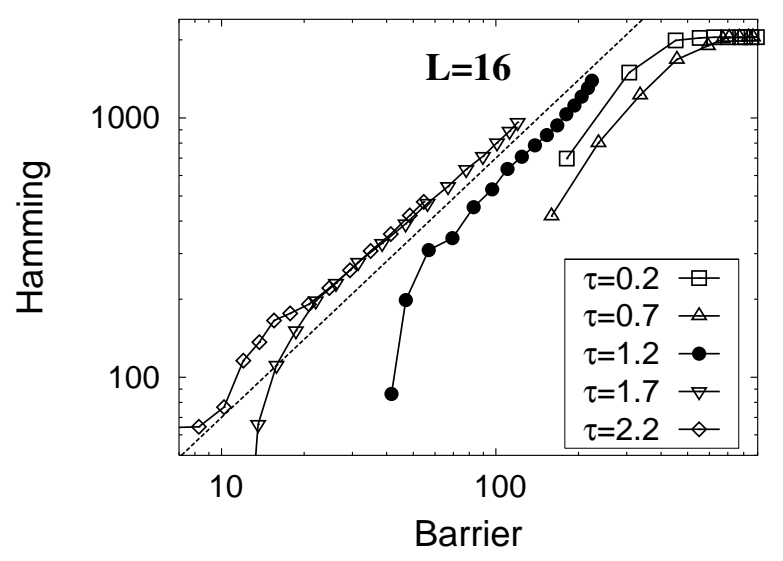

FIG. 7: Plot of the Hamming distance between successive low-energy records as a function of the intervening barrier height, obtained from Figs. 5 ] and 6 by eliminating the valley index dependence between them. The relation for each value of $\tau$ appears to be in fact linear, even for $\tau<1$ before the Hamming distances saturate. Linearity is exemplified by the dashed line of slope 1; the log-log scale was merely chosen for better visibility.

quite linear aside from finite-size effects. Hence, this is all but the first indication of a quantity attributable the landscape geometry itself. The purely geometric origin of this feature is further emphasized by the fact that the data for all $\tau$ very nearly collapses onto a single line, showing only a weak $\tau$-dependence in the slope. While it is not too surprising to obtain such a linear relation from the ratio of two linear relations for the thermal search in Ref. 5, in turn, it is outright amazing to extract a simple linear relation from the ratio of two exponentials in the case of EO here. A linear relationship between Hamming distance and barrier records was obtained long ago for the SK-model [ $\underline{6}$, 7], and was inferred experimentally from thermo-remanent magnetization data [10]. We also note that the largest Hamming distance achievable for a given barrier. as opposed to the typical one, grows exponentially with the barrier in both $2 \mathrm{~d}$ and $3 \mathrm{~d}$ spinglasses [29].

A qualitative snapshot of the difference between consecutive record configurations is provided in Fig 8 There we plot the clusters of overturned spins between two energy records at $n_{V}=9$ and $n_{V}=10$ for some random instance of $L=16$ and various $\tau$. As can be expected from Fig. [6] these records differ by fewer spins for increasing $\tau$. Hence, for low $\tau$, the interfaces between flipped and unflipped spins percolates and is rather indistinct, for $\tau>1$ isolated individual clusters become discernible.

Further quantitative insight into the scope of the search can be obtained by looking at the overlap between earlier-found configurations and those arrived at for later times of the search. To this end, we store the optimal configuration found after one sweep in valley $n_{V}=0$ and measure its overlap with later record energy configurations in valley $n_{V}=k$. This allows us to define a

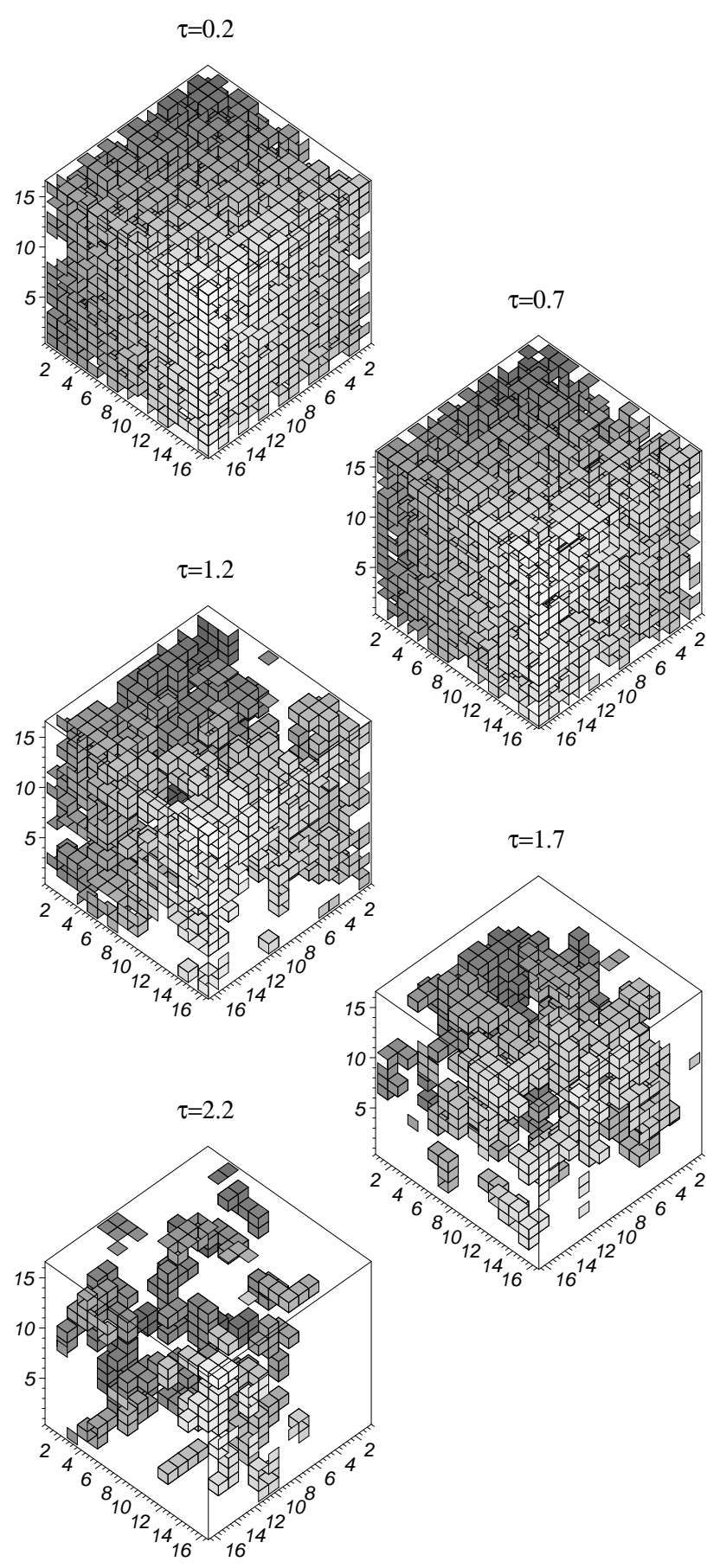

FIG. 8: Snapshot of the clusters of spins which have changed between the configurations of two consecutive energy records at $n_{V}=9$ and 10 found by $\tau$-EO with $\tau=0.2, \ldots, 2.2$, for some instance of $L=16$. Patches cut across bonds along which the relative state of adjacent spins has changed. All surfaces are actually closed and only appear to be open due to the periodic boundary conditions. For small $\tau$, all spins appear to have flipped many times, and it is impossible to tell "inside" from "outside," while for larger $\tau$ a large number of spins clearly have remained "frozen". It appears that for the optimal $\tau=1.2$ flipped spins just about percolate. 


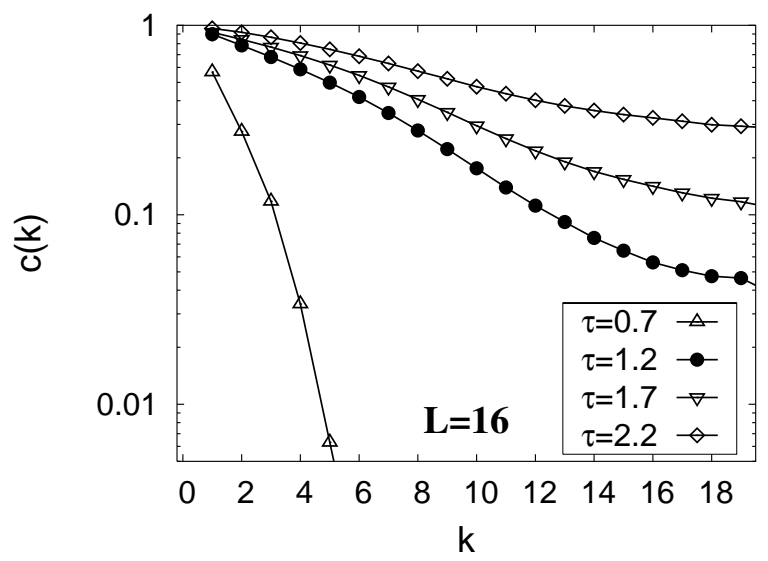

FIG. 9: Plot of the correlations $c(k)$ defined in Eq. 5 between configurations of record energy in valley $n_{V}=k$ and $n_{V}=0$. As in Fig. [6] the $\tau$-dependence here is monotone. The search for small $\tau$ quickly decorrelates, but reaches a plateau value for $\tau>1$.

correlation between $c(k)=c\left(n_{V}=0 ; n_{V}=k\right)$ via the Hamming distance $H(k)$ between them:

$$
c(k)=1-\frac{H(k)}{N / 2} .
$$

Fig. 9 shows that the extremal search decorrelates uniformly faster for decreasing $\tau$, similar to a thermal search for increasing $T$. But for $\tau>1$ the correlations decay about exponentially with the valley index and appear to converge to a plateau value $c_{\infty}$ at large $k$, similar to a low-temperature search. Such a plateau indicates a certain number of "frozen" variables which provide a "backbone" for any near-optimal configuration. In contrast, for $\tau<1$ the extremal search decorrelates almost instantly, and there is only a plateau at $H=N / 2$, the most likely distance for any two random configurations.

Finally, we also look at the hitting time for energy records with a valley, which illuminates their internal structure. If we call $t_{w}$ ("waiting time") the time when a valley was entered, $t_{m}$ the time when the energy minimum was hit, and $t_{x}$ the time when the valley was exited (and the next one entered), then Ref. [5] defined a relative hitting time as

$$
h=\frac{t_{m}-t_{w}}{t_{x}-t_{w}}, \quad 0 \leq h \leq 1,
$$

(called " $\tau$ " in Ref. [5]). For valleys without any structure, the average $h$ would be close to zero, but close to one for highly structured valleys. In Ref. [5] for the thermal search at low temperatures it was found that the distribution for $h$ is peaked at large $h$ values, indicative of the high internal structure of the spin glass valleys. In Fig. 10] we plot the probability density function $G\left(h, t_{w}\right)$ of observing hitting time $h$ in a valley entered at $t_{w}$. We find a strong variation with the parameter $\tau$ in the way $\tau$ EO explores that internal structure. For large $\tau$, the extremal search behaves similar to the lowest-temperature

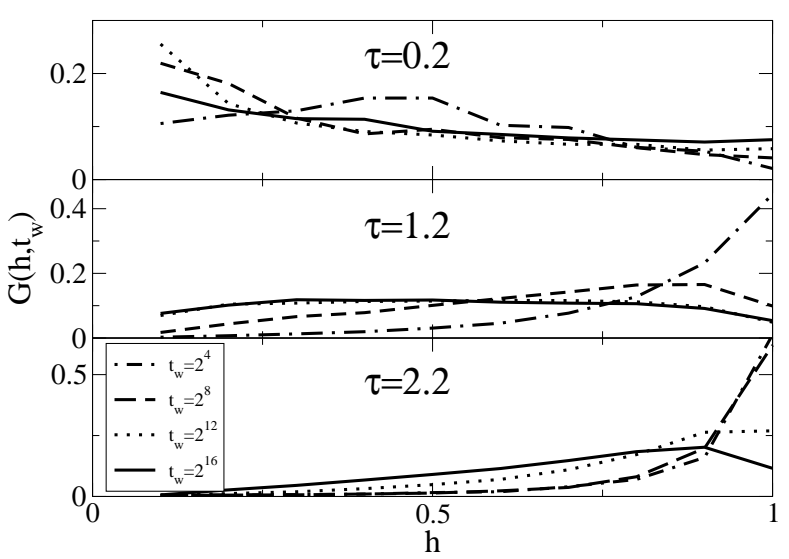

FIG. 10: Plot of the hitting probability $G\left(h, t_{w}\right)$ as a function of the hitting time $h$ defined in Eq. (6) for valleys with increasing entry times $t_{w}$. Shown are the probabilities for $\tau$-EO for $\tau=0.2,1.2$, and 2.2 , all at $L=16$. Note that for $\tau=1.2$ a near-uniform distribution is quickly reached while for small and large $\tau$ small and large hitting times $h$ predominate, resp.

thermal search, i.e. $G$ is right-skewed, while for very small $\tau$, the extremal search seems to ignore the internal structure and discovers what it considers the minimum quite quickly. For the search at $\tau=1.2$, which typically provides the best energy overall, $h$ is almost uniformly distributed over the time spent in the valley, at least for large $t_{w}$.

In Fig. [11 we find that the average hitting time $\langle h\rangle$ decreases for increasing valley index, ever more rapidly for smaller $\tau$, until it saturates. It may appear that hitting onto the record minimum soon after entering a valley may be a good thing for an optimization with a local search. Yet, since $h$ is measured relative to the length of the residence within a valley, small $\langle h\rangle$ here means only that it takes a long time to exit a valley, without ever taking full account of its internal structure. The best compromise in terms of finding energy records (and leaving valleys) quickly seems to be provided by $\tau=1.2$.

\section{B. Varying System Size}

Based on the findings in Sec. IVA it appears that the most favorable behavior of $\tau$-EO from the standpoint of optimization is obtained near $\tau=1.2$. To study the behavior of the extremal search for varying system size in comparison with the thermal search in Ref. [5], we will therefore fix $\tau=1.2$ in this section.

First, we look again at the number of valleys found for increasing runtime. As Fig. 12 shows, the gain in the number of valleys entered increases roughly on a logarithmic timescale. The growth slows at later times, apparently due to system size effects, since the increase becomes more linear, and differences between data less pronounced, for increasing system size. These findings 


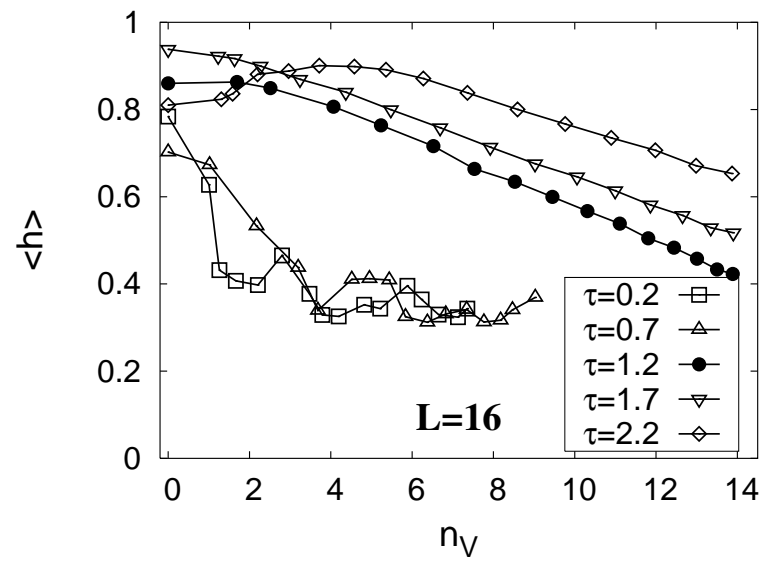

FIG. 11: Plot of the average hitting time $\langle h\rangle$ defined in Eq. [6] as a function of the valley index $n_{V}$. For too small $\tau$, extremal search quickly saturates at short hitting times, unable to leave the current valley. There is a noticeable transition in behavior for $\tau>1$ with a linear decay in $\langle h\rangle$ with a slope that slowly decreases with increasing $\tau$.

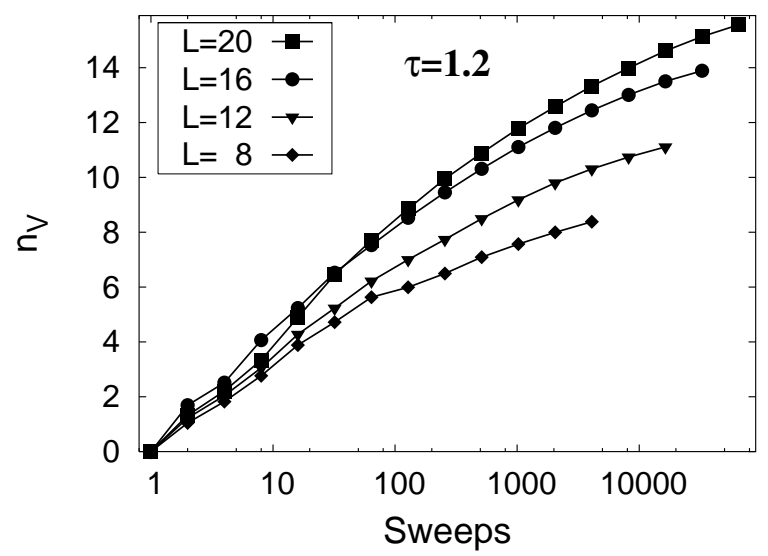

FIG. 12: Plot of the valleys found by EO on a logarithmic timescale at fixed $\tau=1.2$ but for system sizes $L=8,12$, 16 , and 20. Progress toward new valleys occurs linearly on a logarithmic time scale, altered at later times by finite size effects, which become less pronounced for larger $L$.

are very similar to fixed-temperature data (see inset of Fig. 1) in Ref. 5].

After one system sweep from a random initial state, the energy per spin reaches a typical level that strongly depends on $\tau$, as Fig. 4 shows, albeit very little on $L$, according to Fig. 13] But progressing further toward lower energy records through subsequent valleys soon yields diminishing returns. In contrast, for the thermal search in Ref. 5] (see Fig. 4 there) new energy records provide a constant gain $\Delta$ in energy, which in itself depends on $L$ and $T$. It should be noted, though, that the extremal search reaches extremely low energies quickly and becomes sensitive to the presence of the ground state. The behavior of a thermal algorithm is more closely resembled at smaller $\tau$, such as $\tau=0.7$ in Fig. [4 for which the

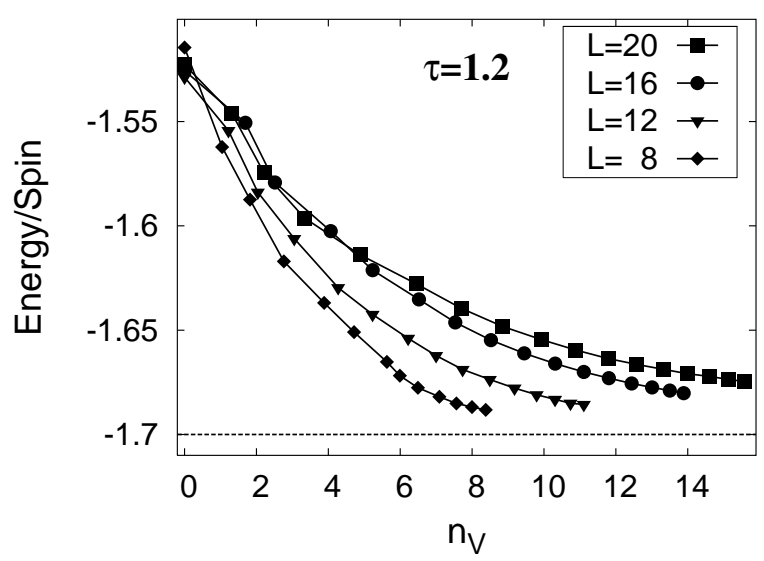

FIG. 13: Plot of the energy records found by EO within a given valley as a function of the valley index $n_{V}$. Here $\tau=$ 1.2 is fixed and system size $L$ is varied. The dashed line at $\approx-1.70$ marks the average ground state energy density for $L \rightarrow \infty$ [28]. Convergence toward this limit at our fixed runtime becomes ever more difficult for increasing $L$. Yet, the saturation at larger $n_{V}$ clearly indicates the proximity of the ground state effecting the search.

decrease in energy scales linearly with $n_{V}$.

As in Fig. 15 for $\tau>1$, Fig. 14 shows that barrier heights scale exponentially for $\tau=1.2$, independent of system size. In fact, that data for different $L$ appears to collapse automatically, without any rescaling. This effect can be explained by the definition of barrier heights as measured relative to the lowest preceding energy record. This would imply that to leave the $i$-th valley an extremal search needs to scale a certain barrier height that is largely insensitive to the system size. (Any deviation from collapse could well be due to a certain arbitrariness in gauging $n_{V}=0$ after a single sweep.) Although on a different scale, the barrier heights for a thermal search were also found to be only weakly dependent on system size (see Fig. 2 in Ref. [5]).

Hamming distances between consecutive energy records are shown in Fig. [15. Similar to the barrier height, the Hamming distance also grows exponentially with the valley index $n_{V}$, showing a significant, but nonextensive, $L$-dependence. The Hamming distances found for $\tau=1.2$ here correspond about to the largest found in the thermal search in Ref. 5] at the highest temperatures, $i$. e. just below $T_{g}$. This demonstrates the breadth of the extremal search for an optimal choice of $\tau$.

Eliminating the valley-index dependence between barrier heights in Fig. 14 and Hamming distances in Fig. 15. as in Fig. 7 we again find a nearly linear relation. This fact re-affirms the purely geometrical origin of this relation, independent of system size.

Finally, in Fig. 17 we show the behavior of the average hitting time $\langle h\rangle$ for different $L$. In each case, $\langle h\rangle$ falls linearly with valley index $n_{V}$ with a size-independent slope. Hence, the data can be collapsed with a simple shift in $n_{V}$. That shift is not uniform in $L$, which could well be 


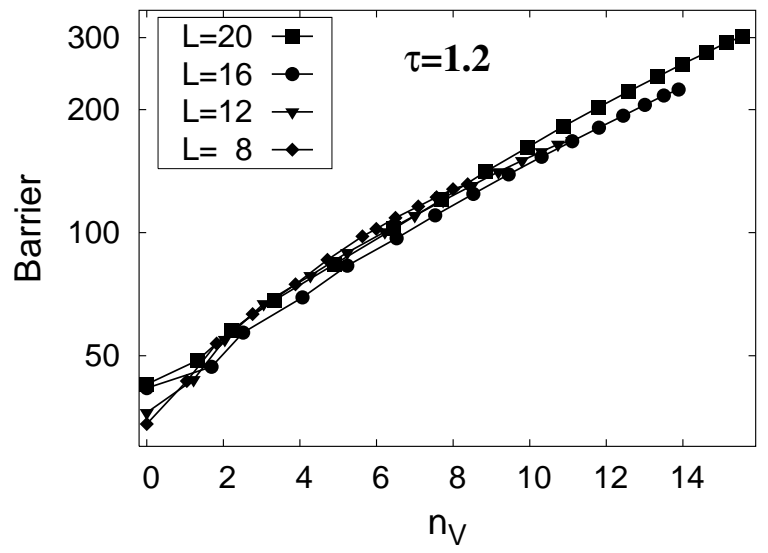

FIG. 14: Plot of the barrier height as a function of valley index $n_{V}$ as in Fig. [5]but for fixed $\tau=1.2$ and different system sizes $L$. Since $\tau>1$, barrier heights rise exponentially. Barriers are measured relative to the most recent low-energy record and appear to be independent of $L$.

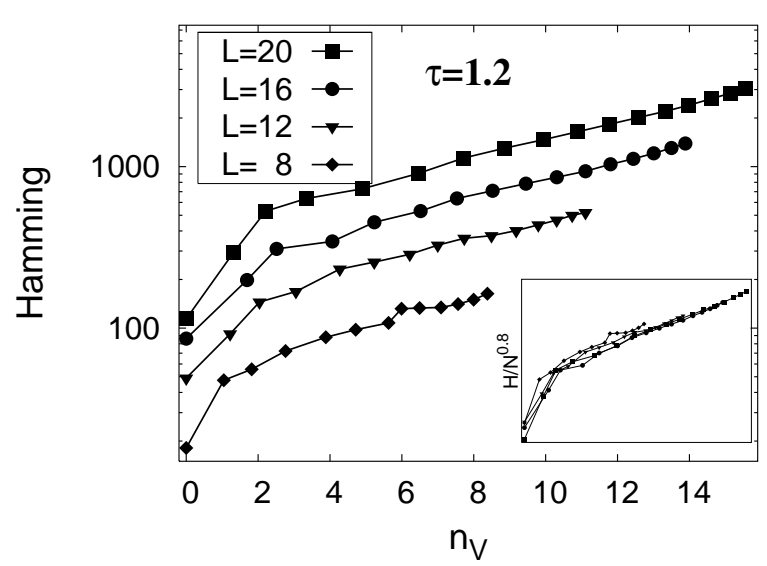

FIG. 15: Plot of the Hamming distance between successive low-energy records as a function of valley index $n_{V}$ at fixed $\tau=1.2$ and variable $L$. The inset shows the same data rescaled by $N^{0.8}$ where $N=L^{3}$.

due to the ambiguity in gauging $n_{V}$.

\section{CONCLUSIONS}

The energy landscape of a spin-glass is probed in this work with a non-thermal optimization algorithm, Extremal Optimization (EO). Energy values of record magnitude are used to partition the states visited into a sequence of valleys, indexed by $n_{V}$. Within each valley the state of lowest energy, or minimum, is the best result obtained with the algorithm on a given time scale. The energy barrier separating two consecutive minima is, by construction, the largest ever barrier scaled on the same time scale. The sole adjustable parameter of EO, $\tau$ and the system size $N=L^{3}$ are both varied systematically in

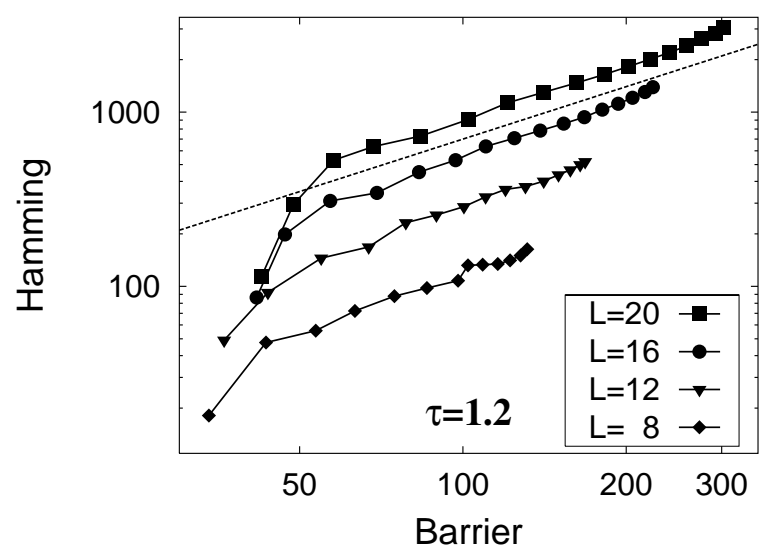

FIG. 16: Plot of the Hamming distance as a function of the barrier height at fixed $\tau=1.2$ and variable $L$. This data was obtained from Figs. 14 and 15] by eliminating the valley-index dependence between them. Linearity is exemplified by the dashed line of slope 1 .

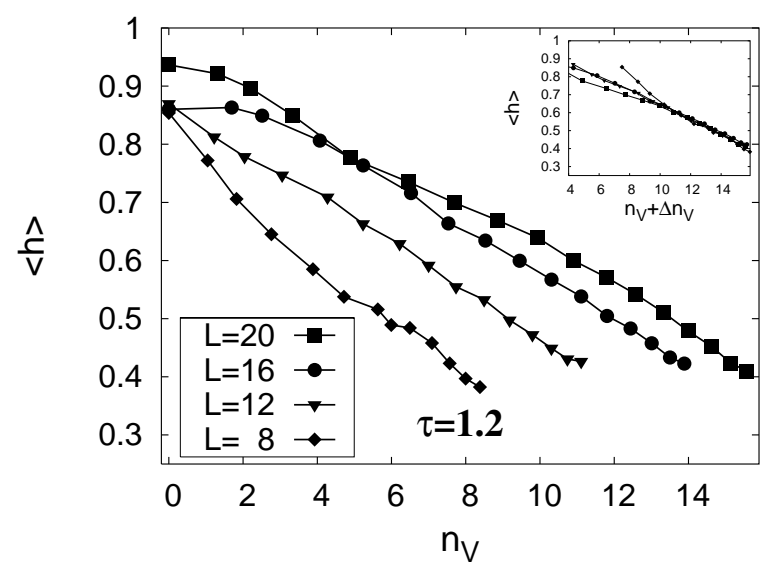

FIG. 17: Plot of the average hitting time $\langle h\rangle$ defined in Eq. (6) as a function of the valley index $n_{V}$ for fixed $\tau=1.2$ and various $L$. Like in Fig. 11] $\langle h\rangle$ falls linearly with $n_{V}$, but with a $L$-independent slope. The inset shows that a simple shift in $n_{V}$ collapses the data for large $n_{V}$ quite well.

the investigations.

Comparing our present results to those of Ref. [5], which uses the same classification scheme in connection with a thermal algorithm, the Waiting Time Algorithm (WTM), we are able to disentangle the intrinsic, or geometrical, properties of the landscape from those specifically linked to the two algorithms. These aspects are separately discussed in this sequel.

Both EO and WTM uncover a non-trivial valley structure whose overall features are broadly similar. The first observation is that, in order to find states of lower energy, progressively higher barriers must be surmounted. The extremal property of these intervening barriers implies that the sequence of lowest minima encountered can (approximately) be treated as a Poisson process with logarithmic time arguments [5]. Such description can only 
work as long as the system remains far away from the global minimum. Indeed, the number $n_{V}$ of valleys explored grows on average in near logarithmic fashion and the decreasing logarithmic slope apparent for the 'best' value of $\tau$ likely reflects the fact that once the system is near to the global optimum, further improvements become harder to achieve.

The magnitude of the highest barrier scaled grows linearly with $n_{V}$ in the WTM analysis, but exponentially with EO. The same is true for the Hamming distance between consecutive minima. By eliminating $n_{V}$, from these exponential relationships a linear dependence emerges, which links the barrier and the Hamming distance between consecutive minima. This concurs with the WTM result, and the linear dependence thus stands out as a geometric property of the energy landscape. For growing system size, and $\tau=1.2$, the Hamming distance between consecutive minima grows as $N^{0.8}$, which is qualitatively similar to the $N^{0.95}$ scaling found with the WTM. Also similar is the decay of the configuration overlap between minima $k$ valleys apart, in both cases nearly exponential in $k$.

The aspect where WTM and EO mostly differ is in the distribution of the time spent searching for the lowest energy state within a fixed valley. Unlike the WTM, EO locates this minimum fairly quickly, at least for values of $\tau$ close to 1.2 , which is the best value in terms of optimization performance. In other words, EO seems to be able to roam more easily through configuration space, which is in accord with the original intention behind its design. Short-ranged spin glasses are probably the test problem where EO is at its worst in terms of optimization performance, yet its performance is slightly better than what WTM can achieve in terms of, say, the lowest energy achieved in a fixed number of updates. However, from a broader optimization perspective the performance of the two algorithms is similar, since lower energy value are logarithmically spaced in time. This, we suggest, might be a general feature of local searches in complex landscapes with a large number of near-equivalent minima.

From a physics point of view, applying a non-thermal algorithm to landscape exploration removes the appeal of using concepts adapted from thermal-equilibrium, such as the free energy, to describe the dynamics. Since, as we have shown, the process of jumping from one valley to a lower-lying valley, is only weakly dependent on the algorithm chosen, thermal concepts are likely to be generally irrelevant for the drift part of the dynamics even though the dynamical update rules obey detailed balance. As we have argued elsewhere [30, 31] the reason for this is that these jumps are effectively irreversible on the time scale at which they occur.

\section{ACKNOWLEDGMENTS}

We would like to thank J. Dall for helpful discussions. This work was partially funded by NSF grant DMR0312510 .
[1] New Optimization Algorithms in Physics, eds. A. K. Hartmann and H. Rieger, (Wiley-VCH, Weinheim, 2004).

[2] Landscape Paradigms in Physics and Biology, eds. H. Frauenfelder et. al. (Elsevier, Amsterdam, 1997).

[3] S. Boettcher and A. G. Percus, Artificial Intelligence 119, 275 (2000).

[4] S. Boettcher and A. G. Percus, Phys. Rev. Lett. 86, 5211 (2001).

[5] J. Dall and P. Sibani, Eur. Phys. J. B 36, 233-243 (2003).

[6] K. Nemoto, J. Phys. A: Math. Gen. 21, L287 (1988). D. Vertechi and M.A. Virasoro, J. Phys. France 50, 23252332 (1989).

[7] D. Vertechi and M.A. Virasoro, J. Phys. France 50, 23252332 (1989).

[8] A. Billoire and E. Marinari, J. Phys. A: Math. Gen. 34, L727-L734 (2001).

[9] P. Sibani and K. H. Hoffmann, Phys. Rev. Lett. 63, 2853 (1989).

[10] M. Lederman, R. Orbach, J.M. Hammann, M. Ocio and E. Vincent, Phys. Rev. B, 44, 7403 (1991)

[11] P. Sibani and K. H. Hoffmann, Europhys. Lett. 16, 423 (1991).

[12] J. P. Bouchaud and D.S. Dean, J. Phys. I France. 5, 265 (1995).

[13] Y. G. Joh, R. Orbach, and J. Hamman, Phys. Rev. Lett. 77, 4648 (1995).
[14] K. H. Hoffmann, S. Schubert, and P. Sibani, Europhys. Lett. 38, 613 (1997).

[15] P. Sibani and K. H. Hoffmann, Physica A 234, 751 (1997).

[16] Y. G. Joh, R. Orbach, G. G. Wood, J. Hammann and E. Vincent, Phys. Rev. Lett. 82, 438 (1999).

[17] A. Crisanti and F. Ritort, Europhys. Lett. 52, 640 (2000).

[18] L. Buisson, L. Bellon, and S. Ciliberto, J. Phys. Cond. Mat. 15, S1163 (2003).

[19] S. Kirkpatrick, C. D. Gelatt, and M. P. Vecchi, Science 220, 671 (1983).

[20] P. Salamon, P. Sibani, and R. Frost, Facts, Conjectures, and Improvements for Simulated Annealing (Society for Industrial \& Applied Mathematics, 2002).

[21] S. Boettcher, J. Math. Phys. A: Math. Gen. 32, 52015211 (1999).

[22] S. Boettcher and A. G. Percus, Physical Review E 69, 066703 (2004).

[23] S. Boettcher, Eur. Phys. J. B 31, 29-39 (2003).

[24] S. Boettcher and M. Grigni, J. Phys. A. 35, 1109 (2002).

[25] S. Boettcher and A. G. Percus, Phys. Rev. E 64, 026114 (2001).

[26] A. A. Middleton, Phys. Rev. E 69, 055701 (R) (2004).

[27] J. Dall and P. Sibani, Computer Physics Communication 141, 260 (2001).

[28] K. F. Pal, Physica A 233, 60-66 (1996). 
[29] P. Sibani, Physica A 258, 249 (1998).

[30] P. Sibani and J. Dall, Europhys. Lett. 64, 8-14 (2003).

[31] P. Sibani and H. J. Jensen, Europhys. Lett. 69, 563-569 